03

\title{
Рассеяние поляризованного и естественного света монослоем сферических однородных пространственно упорядоченных частиц при освещении по нормали
}

\author{
(C) Н.А. Лойко, А.А. Мискевич, В.А. Лойко \\ Институт ффизики им. Б.И. Степанова НАН Беларуси, \\ 220072 Минск, Беларусь \\ e-mail: n.loiko@ifanbel.bas-net.by, miskevic@ifanbel.bas-net.by, loiko@ifanbel.bas-net.by
}

Поступила в редакцию 27.06.2018 г.

Получены выражения для определения оптических характеристик монослоя однородных монодисперсных сферических частиц при освещении по нормали плоской электромагнитной волной с произвольной поляризацией и неполяризованным излучением. Они основаны на квазикристаллическом приближении (ККП) теории многократного рассеяния волн (ТМРВ) и мультипольном разложении полей и тензорной функции Грина по векторным сферическим волновым функциям. Рассмотрено влияние состояния поляризации падающей волны на угловую структуру излучения, рассеянного частично упорядоченным монослоем и монослоем с неидеальной решеткой.

DOI: $10.21883 /$ OS.2018.11.46820.197-18

\section{Введение}

В последние десятилетия активно исследуются монослои частиц, размеры которых сопоставимы с длиной волны оптического излучения, поскольку они имеют большой потенциал применения в оптике, фотонике, опто- и микроэлектронике и многих других областях. Они используются при исследовании, разработке и создании солнечных элементов, светодиодов, фотонных кристаллов, синтетических опалов, антиотражающих покрытий, фильтров пропускания и отражения, диффузных рассеивателей, детекторов, резонаторов, лазеров [1-15].

Наиболее полное описание взаимодействия излучения с монослоем частиц дают методы, учитывающие дифракцию, интерференцию и многократное рассеяние волн. Можно выделить два основных подхода, активно развиваемых и используемых в настоящее время. Первый основан на теории дифракции низкоэнергетических электронов и методе Корринги-Кона-Ростокера [16-20], развитых на случай электромагнитных полей. Он удобен для описания рассеяния света в структурах с идеальными решетками. Второй - на теории многократного рассеяния волн [21-43]. Он обычно используется для моделирования рассеяния и распространения волн в случайных и частично упорядоченных (имеющих ближний пространственный порядок в расположении частиц) дисперсных средах. Его преимущество заключается в статистическом описании структуры ансамбля частиц, что позволяет сравнительно просто моделировать разупорядочение в пространственном положении частиц и создавать эффективные алгоритмы определения оптических характеристик таких систем.

Метод и уравнения для описания когерентной (прямопрошедшей и зеркально отраженной) компоненты излучения, рассеянного частично упорядоченным моно- слоем однородных полидисперсных сферических частиц, освещаемым по нормали плоской циркулярно поляризованной электромагнитной волной, описаны в работе [31]. Они основаны на квазикристаллическом приближении (ККП) [23] теории многократного рассеяния волн и мультипольном разложении электромагнитных полей и тензорной функции Грина. Предложенный в [31] подход использован многими авторами для исследования когерентного пропускания и отражения излучения монослоями частиц с ближним порядком (частично упорядоченными монослоями, рис. 1,a) [1,34-40], радиальная функция распределения [44] частиц в которых описывается в приближении Перкуса-Йевика для твердых сфер [45].

Нами в работах [46,47], предложен метод моделирования радиальной функции распределения частиц в монослое с „неидеальным дальним“ порядком. Такой слой представляет собой двумерный поликристалл, отдельные домены которого имеют неидеальную решетку. Неидеальность состоит в отклонениях центров частиц от положений в узлах идеальной решетки (рис. $1, b)$. Метод позволяет в рамках ККП анализировать оптические характеристики монослоев монодисперсных сферических частиц практически с любым фактором заполнения монослоя. Метод использован при описании когерентного пропускания и отражения излучения трехмерными дисперсными структурами $[11,12,48]$, для восстановления показателя преломления частиц трехмерного фотонного кристалла $[49,50]$, разработки модели солнечного элемента с активным слоем из частиц кремния [13-15].

Метод описания углового распределения излучения, рассеянного монослоем однородных монодисперсных сферических частиц при освещении по нормали плоской циркулярно поляризованной электромагнитной волной, основанный на квазикристаллическом приближении, представлен нами в [51,52]. Рассмотрены характеристики 
$a$

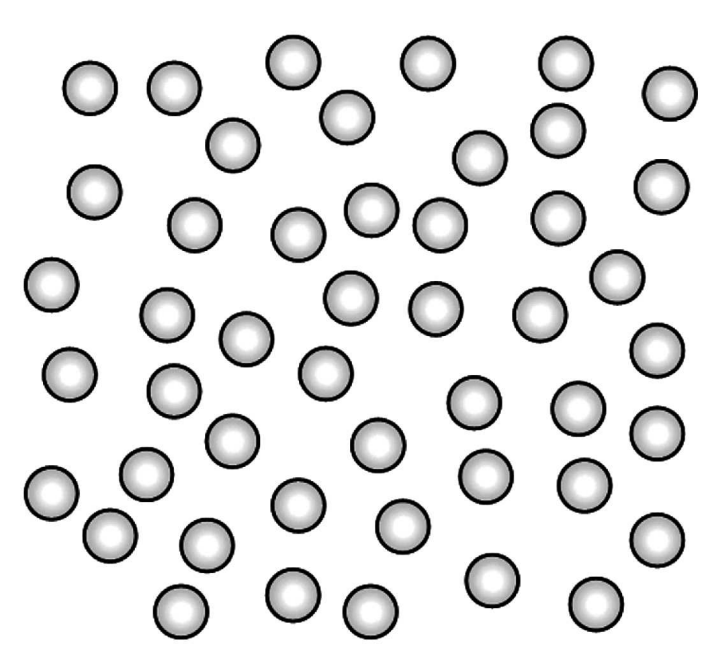

$b$

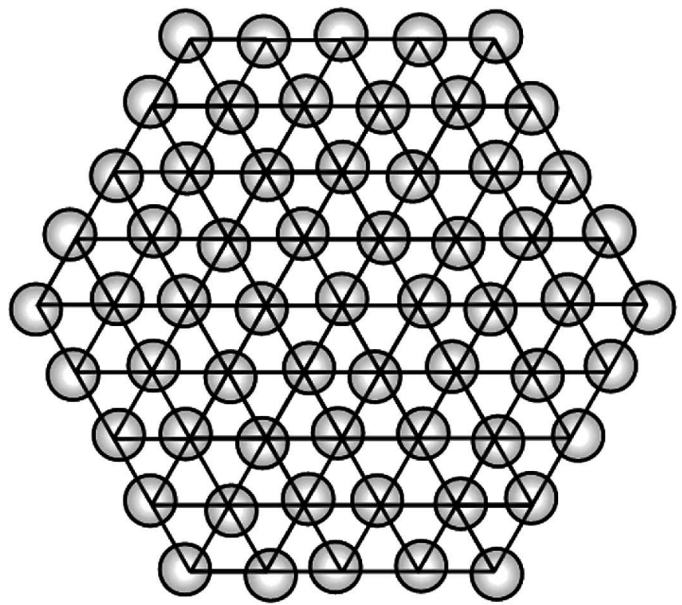

Рис. 1. Схематическое изображение монослоя монодисперсных сферических частиц с ближним порядком в их пространственном расположении (частично упорядоченного монослоя) (a) и монослоя с „неидеальным дальним“ порядком (показано на примере монослоя с неидеальной треугольной решеткой) $(b)$.

рассеяния [51,52] и поглощения [51] излучения частично упорядоченным монослоем и монослоем с неидеальной решеткой. В настоящей работе получены соотношения и приведены результаты расчетов угловой структуры излучения, рассеянного монослоем при освещении плоской электромагнитной волной с произвольной поляризацией.

\section{Основные соотношения}

Рассмотрим монослой, состоящий из $N$ одинаковых сферических частиц, центры которых расположены в плоскости монослоя $(x, y)$ в точках, определяемых радиусами-векторами $\mathbf{R}_{1}, \mathbf{R}_{2}, \ldots, \mathbf{R}_{N}$ относительно начала координат, находящегося в центре произвольно выбранной частицы (рис. 2). Монослой освещается по нормали (вдоль оси $z$ ) плоской электромагнитной волной с электрическим вектором $\mathbf{E}_{0}$ и вектором поляризации $\hat{\varepsilon}_{0}$ :

$$
\mathbf{E}_{0}=\hat{\varepsilon}_{0} e^{i k z},
$$

где $k=2 \pi / \lambda-$ волновое число, $\lambda-$ длина волны падающего излучения. Полагаем, что $\left|\mathbf{E}_{0}\right|=\left|\hat{\varepsilon}_{0}\right|=1$.

Полное поле $\mathbf{E}(\mathbf{r})$ в некоторой точке $\mathbf{r}$ является суммой поля падающей волны и полей волн, рассеянных данной конфигурацией ансамбля частиц (данное расположение частиц $\left.\mathbf{R}_{1}, \mathbf{R}_{2}, \ldots, \mathbf{R}_{N}\right)$ :

$$
\mathbf{E}(\mathbf{r})=\mathbf{E}_{0}(\mathbf{r})+\sum_{j=1}^{N} \mathbf{F}_{j}\left(\mathbf{r}, \mathbf{R}_{j}\right)
$$

Здесь

$$
\mathbf{F}_{j}\left(\mathbf{r}, \mathbf{R}_{j}\right)=\frac{\left(m^{2}-1\right) k^{2}}{4 \pi} \int_{V_{p}} d \mathbf{r}^{\prime} \overleftrightarrow{G}\left(\mathbf{r}, \mathbf{R}_{j}+\mathbf{r}^{\prime}\right) \cdot \mathbf{E}\left(\mathbf{R}_{j}+\mathbf{r}^{\prime}\right)
$$

- поле, создаваемое в точке $\mathbf{r}$ частицей, центр которой находится в точке $\mathbf{R}_{j}, m=n+i \kappa-$ комплексный показатель преломления частицы относительно окружающей среды (относительный комплексный показатель преломления частицы), $V_{p}$ - объем частицы, $0 \leq\left|\mathbf{r}^{\prime}\right| \leq D / 2$, $D$ - диаметр частицы, $\mathbf{E}\left(\mathbf{R}_{j}+\mathbf{r}^{\prime}\right)$ - поле в точке $\mathbf{R}_{j}+\mathbf{r}^{\prime}$ внутри частицы (внутреннее поле), $\overleftrightarrow{G}$ - диадная (тензорная) функция Грина:

$$
\overleftrightarrow{G}\left(\mathbf{r}, \mathbf{r}_{0}\right)=\left[\overleftrightarrow{I}+\frac{1}{k^{2}} \nabla \otimes \nabla\right] \frac{e^{i k\left|\mathbf{r}-\mathbf{r}_{0}\right|}}{\left|\mathbf{r}-\mathbf{r}_{0}\right|}
$$

$\overleftrightarrow{I}$ - единичная диада.

Усреднение уравнения (2) по возможным конфигурациям ансамбля частиц в рамках ККП приводит к следующему уравнению для усредненного поля вне частиц $\langle\mathbf{E}(\mathbf{r})\rangle[31,51,52]$ :

$$
\begin{aligned}
& \langle\mathbf{E}(\mathbf{r})\rangle=\mathbf{E}_{0}(\mathbf{r})+\sum_{j=1}^{N}\left\langle\mathbf{F}_{j}(\mathbf{r}, \mathbf{R})\right\rangle=\mathbf{E}_{0}(\mathbf{r}) \\
& +\frac{\left(m^{2}-1\right) k^{2}}{4 \pi} \rho_{0} \int d \mathbf{R} \int_{V_{p}} d \mathbf{r}^{\prime} \overleftrightarrow{G}\left(\mathbf{r}, \mathbf{R}+\mathbf{r}^{\prime}\right) \cdot\left\langle\mathbf{E}\left(\mathbf{r}^{\prime}\right)\right\rangle_{1},
\end{aligned}
$$

где $\left\langle\mathbf{E}\left(\mathbf{r}^{\prime}\right)\right\rangle_{1}$ - поле в частице, центр которой находится в начале координат, усредненное по положениям остальных частиц (усредненное внутреннее поле). Оно 


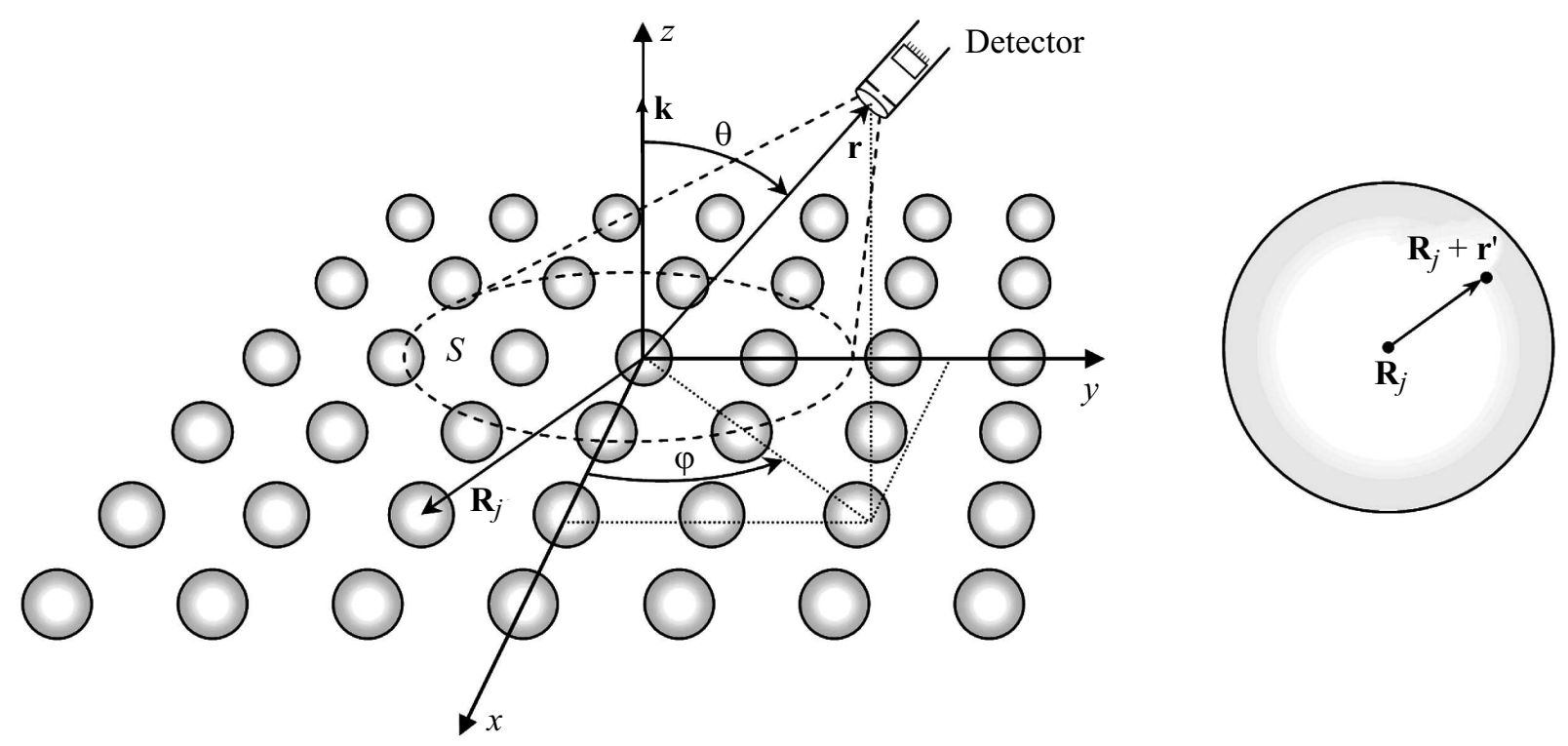

Рис. 2. Схематическое изображение монослоя сферических частиц и геометрии освещения, рассеяния и регистрации рассеянного излучения в дальней зоне монослоя (слева) и отдельной частицы монослоя (справа). $x, y, z$ - лабораторная система координат, связанная с монослоем, начало координат находится в центре частицы, $(x, y)-$ плоскость монослоя, $\mathbf{k}-$ волновой вектор падающей волны (освещение по нормали к монослою вдоль оси $z$ ), $\mathbf{R}_{j}-$ радиус-вектор, описывающий положение центра $j$-й частицы в плоскости монослоя, $\mathbf{R}_{j}+\mathbf{r}^{\prime}-$ радиус-вектор элементарного объема внутри $j$-й частицы, $\mathbf{r}-$ радиус-вектор точки наблюдения, $\theta$ и $\varphi-$ полярный и азимутальный углы рассеяния, $S-$ площадь поверхности монослоя, „видимая“ приемником излучения.

определяется из уравнения [51,52]

$$
\begin{aligned}
& \left\langle\mathbf{E}\left(\mathbf{r}^{\prime}\right)\right\rangle_{1}=\mathbf{E}_{0}\left(\mathbf{r}^{\prime}\right)+\frac{\left(m^{2}-1\right) k^{2}}{4 \pi} \int_{V_{p}} d \mathbf{r}^{\prime \prime} \overleftrightarrow{G}\left(\mathbf{r}^{\prime}, \mathbf{r}^{\prime \prime}\right) \cdot\left\langle\mathbf{E}\left(\mathbf{r}^{\prime \prime}\right)\right\rangle_{1} \\
& +\frac{\left(m^{2}-1\right) k^{2}}{4 \pi} \rho_{0} \int d \mathbf{R} g(R) \int_{V_{p}} d \mathbf{r}^{\prime \prime} \overleftrightarrow{G}\left(\mathbf{r}^{\prime}, \mathbf{R}+\mathbf{r}^{\prime \prime}\right) \cdot\left\langle\mathbf{E}\left(\mathbf{r}^{\prime \prime}\right)\right\rangle_{1} .
\end{aligned}
$$

Уравнения (5) и (6) получены в предположении, что в слое реализуется однородное распределение частиц, и, как следствие, усредненные внутренние поля для всех частиц одинаковы в случае освещения монослоя по нормали. Здесь $\rho_{0}-$ средняя плотность числа частиц (концентрация), $g(R)$ - радиальная функция распределения [44], описывающая вероятность нахождения центра частицы на расстоянии $R$ относительно частицы, центр которой находится в начале координат, $R=|\mathbf{R}|$.

B работах [51,52] приведен детальный вывод решения уравнения (5) для дальней зоны монослоя $\quad\left(\left|\mathbf{r}-\left(\mathbf{R}+\mathbf{r}^{\prime}\right)\right| \gg \lambda\right) \quad$ в предположении, что $\lim _{R \rightarrow \infty} \frac{R}{r}=$ const $\ll 1(r=|\mathbf{r}|)$ (рис. 2). Показано, что как

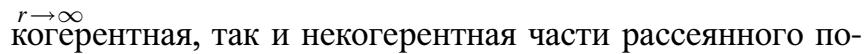
ля определяются амплитудной функцией рассеяния $\mathbf{f}(\hat{\mathbf{r}})$ в направлении $\hat{\mathbf{r}}$ для отдельной частицы монослоя:

$$
\mathbf{f}(\hat{\mathbf{r}})=\frac{\left(m^{2}-1\right) k^{3}}{4 \pi} \int_{V_{p}} d \mathbf{r}^{\prime}(\overleftrightarrow{I}-\hat{\mathbf{r}} \otimes \hat{\mathbf{r}}) e^{-i k \hat{\mathbf{r}} \cdot \mathbf{r}^{\prime}} \cdot\left\langle\mathbf{e}\left(\mathbf{R}^{\prime}\right)\right\rangle_{1}
$$

В соответствии с уравнением (6) $\left\langle\mathbf{E}\left(\mathbf{r}^{\prime}\right)\right\rangle_{1}$ и, следовательно, $\mathbf{f}(\hat{\mathbf{r}})$ учитывают вклад всех частиц монослоя в формирование внутреннего поля рассматриваемой частицы (т.е. многократное рассеяние волн в монослое).

Решения для когерентно прошедшей $\left\langle\mathbf{E}_{t}(\mathbf{z})\right\rangle$ монослой и когерентно (зеркально) отраженной $\left\langle\mathbf{E}_{r}(\mathbf{z})\right\rangle$ волн имеют следующий вид:

$$
\begin{gathered}
\left\langle\mathbf{E}_{t}(\mathbf{z})\right\rangle=\mathbf{E}_{0}(\mathbf{z})+\rho_{0} \frac{2 \pi i}{k^{2}} \mathbf{f}(\hat{\mathbf{z}}) e^{i k|\mathbf{z}|} \\
\left\langle\mathbf{E}_{r}(\mathbf{z})\right\rangle=\rho_{0} \frac{2 \pi i}{k^{2}} \mathbf{f}(-\hat{\mathbf{z}}) e^{i k|\mathbf{z}|}
\end{gathered}
$$

Некогерентная часть рассеянного излучения, возникающая за счет флуктуаций положения частиц, проявляется в интенсивности

$$
I_{i n c}(\mathbf{r})=\mathbf{f}(\hat{\mathbf{r}}) \cdot \mathbf{f}^{*}(\hat{\mathbf{r}}) \frac{\eta}{\pi x^{2}} \frac{S}{r^{2}} S_{2}(2 x \sin \theta) .
$$

Здесь $\eta-$ фактор заполнения монослоя (отношение площади проекций всех частиц на плоскость монослоя к площади, на которой они распределены), $x=\pi D / \lambda-$ параметр дифракции частицы, $D$ - диаметр частицы, $\theta$ - полярный угол рассеяния, $S$ - „видимая“ приемником площадь монослоя, величина которой зависит от угла приема и точки наблюдения (рис. 2), $S_{2}(2 x \sin \theta)-$ двумерный (2D) структурный фактор. Он характеризует влияние пространственного распределения частиц на 
угловую структуру рассеянного излучения [44,53]:

$$
S_{2}(2 x \sin \theta)=1+8 \eta \int_{0}^{\infty}[g(u)-1] J_{0}(2 x u \sin \theta) u d u .
$$

В формуле (11) $u=R / D-$ безразмерная переменная интегрирования, определяющая расстояние в плоскости монослоя, выраженное в диаметрах частиц, $J_{0}(z)-$ цилиндрическая функция Бесселя нулевого порядка.

Для определения амплитудной функции рассеяния $\mathbf{f}(\hat{\mathbf{r}})$ используем метод разложения всех функций, входящих в уравнения (6), (7), по векторным сферическим волновым функциям (ВСВФ) [54,55]. Разложение падающего поля для произвольного вектора поляризации $\hat{\varepsilon}_{0}$ падающей волны (1) получим на основе разложения по ВСВФ матричной функции $\overleftrightarrow{I} e^{-i k z}$. Если записать вектор $\hat{\varepsilon}_{0}$ через его компоненты в декартовых координатах $(x, y)$ :

$$
\hat{\varepsilon}_{0}=\left(\varepsilon_{x} \hat{\mathbf{x}}+\varepsilon_{y} \hat{\mathbf{y}}\right)
$$

To

$$
\begin{aligned}
\mathbf{E}_{0}(\mathbf{r})= & \sum_{l=1}^{\infty} i^{l} \frac{2 l+1}{l(l+1)}\left\{\varepsilon_{x}\left[\mathbf{M}_{o 1 l}^{(1)}(k \mathbf{r})-i \mathbf{N}_{e 1 l}^{(1)}(k \mathbf{r})\right]\right. \\
& \left.-\varepsilon_{y}\left[\mathbf{M}_{e 1 l}^{(1)}(k \mathbf{r})+i \mathbf{N}_{o 1 l}^{(1)}(k \mathbf{r})\right]\right\} .
\end{aligned}
$$

Здесь индексы $e$ и $o$ означают соответственно четную и нечетную функции, определяющие комплексные волновые функции

$$
\begin{gathered}
\mathbf{M}_{1 l}^{(1)}=\mathbf{M}_{e 1 l}^{(1)}+i \mathbf{M}_{o 1 l}^{(1)}, \\
\mathbf{N}_{1 l}^{(1)}=\mathbf{N}_{e 1 l}^{(1)}+i \mathbf{N}_{o 1 l}^{(1)},
\end{gathered}
$$

Представим усредненное поле в частице аналогичным разложением с неизвестными коэффициентами $a_{l M}^{(o, e)}$ и $a_{l E}^{(o, e)}$ :

$$
\begin{aligned}
\left\langle\mathbf{E}\left(\mathbf{r}^{\prime}\right)\right\rangle_{1}= & \sum_{l=1}^{\infty}\left[a_{l M}^{(e)} \mathbf{M}_{e 1 l}^{(1)}(m k \mathbf{r})+i a_{l M}^{(o)} \mathbf{M}_{o 1 l}^{(1)}(m k \mathbf{r})\right. \\
& \left.+a_{l E}^{(e)} \mathbf{N}_{e 1 l}^{(1)}(m k \mathbf{r})+i a_{l E}^{(o)} \mathbf{N}_{o 1 l}^{(1)}(m k \mathbf{r})\right]
\end{aligned}
$$

Для интегрирования (7) разложим по ВСВФ матричную функцию $(\overleftrightarrow{I}-\hat{\mathbf{r}} \otimes \hat{\mathbf{r}}) e^{-i k \hat{\mathbf{r}} \cdot \mathbf{r}^{\prime}}[54,55]$. Используя ортогональность векторных сферических гармоник, получим

$$
\mathbf{f}(\hat{\mathbf{r}})=f_{\theta}(\hat{\mathbf{r}}) \hat{\boldsymbol{\theta}}+f_{\varphi}(\hat{\mathbf{r}}) \hat{\boldsymbol{\varphi}},
$$

где

$$
\begin{aligned}
f_{\theta}(\hat{\mathbf{r}})= & i \sum_{l=1}^{\infty} \frac{(2 l+1)}{l(l+1)}\left[\pi_{l}^{(1)}(\mu)\left(b_{l M}^{(o)} \cos \varphi+i b_{l M}^{(e)} \sin \varphi\right)\right. \\
& \left.+\tau_{l}^{(1)}(\mu)\left(b_{l E}^{(e)} \cos \varphi+i b_{l E}^{(o)} \sin \varphi\right)\right],
\end{aligned}
$$

$$
\begin{aligned}
f_{\phi}(\hat{\mathbf{r}})= & -\sum_{l=1}^{\infty} \frac{(2 l+1)}{l(l+1)} \\
& \times\left[\tau_{l}^{(1)}(\mu)\left(b_{l M}^{(e)} \cos \varphi+i b_{l M}^{(o)} \sin \varphi\right)\right. \\
& \left.+\pi_{l}^{(1)}(\mu)\left(b_{l E}^{(o)} \cos \varphi+i b_{l E}^{(e)} \sin \varphi\right)\right],
\end{aligned}
$$

Угловые функции $\pi_{l}^{(1)}(\mu)=P_{l}^{(1)}(\cos \theta) / \sin \theta, \tau_{l}^{(1)}(\mu)=$ $=d P_{l}^{(1)}(\cos \theta) / d \theta$, где $\mu=\cos \theta, P_{l}^{(1)}(\cos \theta)$ - присоединенные функции Лежандра, $\hat{\boldsymbol{\theta}}$ и $\hat{\boldsymbol{\varphi}}-$ единичные векторы в направлениях, определяемых полярным $\theta$ и азимутальным $\varphi$ углами рассеяния (рис. 2). Коэффициенты разложения $b_{l M}^{(o, e)}$ и $b_{l E}^{(o, e)}$, учитывающие многократное рассеяние волн, связаны с коэффициентами $a_{l M}^{(o, e)}$ и $a_{l E}^{(o, e)}$, определяющими усредненное внутреннее поле (16), следующим образом:

$$
b_{l M}^{(o, e)}=\frac{\left(\psi_{l}^{\prime} \tilde{\psi}_{l}-m \psi_{l} \tilde{\psi}_{l}^{\prime}\right) l(l+1)}{m i^{l}(2 l+1)} a_{l M}^{(o, e)},
$$

$$
b_{l E}^{(o, e)}=\frac{\left(m \psi_{l}^{\prime} \tilde{\psi}_{l}-\psi_{l} \tilde{\psi}_{l}^{\prime}\right) l(l+1)}{m i^{l}(2 l+1)} a_{l E}^{(o, e)} .
$$

Здесь $\psi_{l}=x j_{l}(x), \tilde{\psi}_{l}=m x j_{l}(m x), j_{l}-$ сферическая функция Бесселя $l$-го порядка.

При $\hat{\mathbf{r}}= \pm \hat{\mathbf{z}}$ выражения (18), (19) упрощаются

$$
\begin{aligned}
& \mathbf{f}( \pm \hat{\mathbf{z}})=\mathrm{f}_{x}( \pm \hat{\mathbf{z}}) \hat{\mathbf{x}}+\mathrm{f}_{y}( \pm \hat{\mathbf{z}}) \hat{\mathbf{y}} \\
& \quad=\frac{i}{2} \sum_{l=1}^{\infty}( \pm 1)^{l}(2 l+1)\left[\hat{\mathbf{x}}\left(b_{l M}^{(o)} \pm b_{l E}^{(e)}\right)+i \hat{\mathbf{y}}\left(b_{l M}^{(e)} \pm b_{l E}^{(o)}\right)\right]
\end{aligned}
$$

Здесь $\mathrm{f}_{x}$ и $\mathrm{f}_{y}$ - компоненты векторной амплитудной функции рассеяния, описывающие компоненты рассеянного излучения, поляризованные вдоль осей $x$ и $y$ соответственно. Коэффициенты когерентного пропускания $T_{c}$ и отражения $R_{c}$ монослоя получим, используя формулы (8), (9) и (22),

$$
\begin{gathered}
T_{c}=\left|\mathbf{E}_{l}\right|^{2} /\left|\mathbf{E}_{0}\right|^{2}=\left|\varepsilon_{x}-\frac{\eta}{x^{2}} \sum_{l=1}^{\infty}(2 l+1)\left(b_{l M}^{(o)}+b_{l E}^{(e)}\right)\right|^{2} \\
+\left|\varepsilon_{y}-i \frac{\eta}{x^{2}} \sum_{l=1}^{\infty}(2 l+1)\left(b_{l M}^{(e)}+b_{l E}^{(o)}\right)\right|^{2} \\
R_{c}=\left(\frac{\eta}{x^{2}}\right)^{2}\left[\left|\sum_{l=1}^{\infty}(-1)^{l}(2 l+1)\left(b_{l M}^{(o)}-b_{l E}^{(e)}\right)\right|^{2}\right. \\
\left.+\left|\sum_{l=1}^{\infty}(-1)^{l}(2 l+1)\left(b_{l M}^{(e)}-b_{l E}^{(o)}\right)\right|^{2}\right]
\end{gathered}
$$


Коэффициент некогерентного рассеяния $F_{\text {inc }}$ определим как интеграл по телесному углу $4 \pi$ отношения интенсивности излучения $I_{\text {inc }}(\mathbf{r})$ (формула (10)), peгистрируемой с „видимой“ приемником площади $S$, к интенсивности излучения, падающего на эту площадь $\left(I_{i n c}^{r d}=I_{\text {inc }}(\mathbf{r}) /\left|\mathbf{E}_{0}\right|^{2} S\right)$ :

$$
F_{\mathrm{inc}}=\int_{4 \pi} I_{i n c}^{r d}(\mathbf{r}) r^{2} \sin \theta d \varphi d \theta,
$$

где приведенная интенсивность $I_{i n c}^{r d}$ некогерентно рассеянного монослоем излучения

$$
\begin{aligned}
& I_{i n c}^{r d}(\hat{\mathbf{r}}) r^{2}=I_{i n c}^{r d}(\theta, \varphi)=\frac{\eta S_{2}(2 x \sin \theta)}{\pi x^{2}}\left\{\left|\mathrm{f}_{\theta}(\hat{\mathbf{r}})\right|^{2}+\left|\mathrm{f}_{\varphi}(\hat{\mathbf{r}})\right|^{2}\right\} \\
& =\frac{\eta S_{2}(2 x \sin \theta)}{\pi x^{2}}\left\{\mid \sum_{l=1}^{\infty} \frac{(2 l+1)}{l(l+1)}\right. \\
& \times\left[\pi_{l}^{(1)}(\mu)\left(b_{l M}^{(o)} \cos \varphi+i b_{l M}^{(e)} \sin \varphi\right)\right. \\
& \left.+\tau_{l}^{(1)}(\mu)\left(b_{l E}^{(e)} \cos \varphi+i b_{l E}^{(o)} \sin \varphi\right)\right]\left.\right|^{2} \\
& +\mid \sum_{l=1}^{\infty} \frac{(2 l+1)}{l(l+1)}\left[\tau_{l}^{(1)}(\mu)\left(b_{l M}^{(e)} \cos \varphi+i b_{l M}^{(o)} \sin \varphi\right)\right. \\
& \left.\left.+\pi_{l}^{(1)}(\mu)\left(b_{l E}^{(o)} \cos \varphi+i b_{l E}^{(e)} \sin \varphi\right)\right]\left.\right|^{2}\right\} .
\end{aligned}
$$

При $\hat{\mathbf{r}}= \pm \hat{\mathbf{z}}$ эта формула преобразуется в соответствии c (22)

$$
\begin{aligned}
& I_{i n c}^{r d}( \pm \hat{\mathbf{z}}) z^{2}=\frac{\eta S_{2}(0)}{\pi x^{2}}\left\{\left|\mathrm{f}_{x}( \pm \hat{\mathbf{z}})\right|^{2}+\left|\mathrm{f}_{y}( \pm \hat{\mathbf{z}})\right|^{2}\right\} \\
& =\frac{\eta S_{2}(0)}{4 \pi x^{2}}\left\{\left|\sum_{l=1}^{\infty}( \pm 1)^{l}(2 l+1)\left(b_{l M}^{(o)} \pm b_{l E}^{(e)}\right)\right|^{2}\right. \\
& \left.+\left|\sum_{l=1}^{\infty}( \pm 1)^{l}(2 l+1)\left(b_{l M}^{(e)} \pm b_{l E}^{(o)}\right)\right|^{2}\right\} .
\end{aligned}
$$

Для определения коэффициентов $b_{l M}^{(o, e)}$ и $b_{l E}^{(o, e)}$ применим к уравнению (6) для усредненного поля в частице метод, подобный описанному в работе [31]. Для этого выразим четные и нечетные волновые функции, входящие в выражения для падающего поля (13) и для усредненного поля в частице (16), через сумму и разность комплексно сопряженных волновых функций (14), (15):

$$
\begin{aligned}
\mathbf{M}_{e 1 l}^{(1)} & =\frac{1}{2}\left(\mathbf{M}_{1 l}^{(1)} \pm \mathbf{M}_{1 l}^{(1) *}\right), \\
\mathbf{N}_{\substack{e 1 l \\
o 1 l}}^{(1)} & =\frac{1}{2}\left(\mathbf{N}_{1 l}^{(1)} \pm \mathbf{N}_{1 l}^{(1) *}\right) .
\end{aligned}
$$

Здесь верхний знак „+“ соответствует функциям $\mathbf{M}_{e 1 l}^{(1)}$, $\mathbf{N}_{e 1 l}^{(1)}$, а нижний знак „,-“ функциям $\mathbf{M}_{o 1 l}^{(1)}, \mathbf{N}_{o 1 l}^{(1)}$.

Разложим функции Грина по векторным сферическим волновым функциям и используем теорему сложения для функций с аргументом $\left(\mathbf{R}+\mathbf{r}^{\prime}\right)$ в соответствии с формулами, приведенными в $[31,56]$. Приравнивая коэффициенты при одинаковых ВСВФ, получим алгебраические уравнения для сумм

$$
b_{l M(E)}^{(s)}=b_{l M(E)}^{(o)}+b_{l M(E)}^{(e)}
$$

и разностей

$$
b_{l M}^{(d)}=b_{l M}^{(o)}-b_{l M}^{(e)}, \quad b_{l E}^{(d)}=b_{l E}^{(e)}-b_{l E}^{(o)}
$$

искомых коэффициентов $b_{l M}^{(o, e)}$ и $b_{l E}^{(o, e)}$ :

$$
\begin{gathered}
\left\{\begin{array}{c}
b_{l M}^{(s)}=c_{l M}\left[\varepsilon_{x}-i \varepsilon_{y}+8 \eta \sum_{l^{\prime}} A_{l l^{\prime}} b_{l^{\prime} M}^{(s)}+B_{l l^{\prime}} b_{l^{\prime} E}^{(s)}\right] \\
b_{l E}^{(s)}=c_{l E}\left[\varepsilon_{x}-i \varepsilon_{y}+8 \eta \sum_{l^{\prime}} A_{l l^{\prime}} b_{l^{\prime} E}^{(s)}+B_{l l^{\prime}} b_{l^{\prime} M}^{(s)}\right]
\end{array}\right. \\
\left\{\begin{array}{l}
b_{l M}^{(d)}=c_{l M}\left[\varepsilon_{x}+i \varepsilon_{y}+8 \eta \sum_{l^{\prime}}(-1)^{l^{\prime}-l}\left[A_{l l^{\prime}} b_{l^{\prime} M}^{(d)}-B_{l l^{\prime}} b_{l^{\prime} E}^{(d)}\right]\right] \\
b_{l E}^{(d)}=c_{l E}\left[\varepsilon_{x}+i \varepsilon_{y}+8 \eta \sum_{l^{\prime}}(-1)^{l^{\prime}-l}\left[A_{l l^{\prime}} b_{l^{\prime} E}^{(d)}-B_{l l^{\prime}} b_{l^{\prime} M}^{(d)}\right]\right]
\end{array}\right.
\end{gathered}
$$

В уравнениях (32),(33) коэффициенты $A_{l l^{\prime}}$ и $B_{l l^{\prime}}$ определяются по следующим формулам:

$$
\begin{aligned}
& A_{l l^{\prime}}=\frac{2 l^{\prime}+1}{2}\left[l(l+1) l^{\prime}\left(l^{\prime}+1\right)\right]^{-1 / 2} \\
& \times \sum_{p=0,2, \ldots}(-1)^{p / 2}(2 p+1)\left[l(l+1)+l^{\prime}\left(l^{\prime}+1\right)-p(p+1)\right] \\
& \times P_{p}(0)\left(\begin{array}{lll}
l & l^{\prime} & p \\
0 & 0 & 0
\end{array}\right)\left(\begin{array}{ccc}
l & l^{\prime} & p \\
1 & -1 & 0
\end{array}\right) H_{p}, \\
& B_{l l^{\prime}}=\frac{2 l^{\prime}+1}{2}\left[l(l+1) l^{\prime}\left(l^{\prime}+1\right)\right]^{-1 / 2} \\
& \left.\times \sum_{p=0,2, \ldots}\left(\begin{array}{ccc}
-1)^{p / 2}(2 p+1) \\
\times\left[( p + l - l ^ { \prime } ) ( p - l + l ^ { \prime } ) \left(l+l^{\prime}+1+p\right.\right.
\end{array}\right)\left(l+l^{\prime}+1-p\right)\right]^{1 / 2} \\
& \times P_{p}(0)\left(\begin{array}{ccc}
l & l^{\prime} & p-1 \\
0 & 0 & 0
\end{array}\right)\left(\begin{array}{ccc}
l & l^{\prime} & p \\
1 & -1 & 0
\end{array}\right) H_{p},
\end{aligned}
$$

где

$$
H_{p}=\int_{1}^{\infty} g(u) h_{p}^{(1)}(2 x u) u d u .
$$


Коэффициенты $c_{l E}$ и $c_{l M}-$ коэффициенты Ми для рассеянного поля изолированной частицы:

$$
\left\{\begin{array}{l}
c_{l E}=\frac{m \psi_{l}(m x) \psi_{l}^{\prime}(x)-\psi_{l}(x) \psi_{l}^{\prime}(m x)}{m \psi_{l}(m x) \chi_{l}^{\prime}(x)-\chi_{l}(x) \psi_{l}^{\prime}(m x)}, \\
c_{l M}=\frac{\psi_{l}(m x) \psi_{l}^{\prime}(x)-m \psi_{l}(x) \psi_{l}^{\prime}(m x)}{\psi_{l}(m x) \chi_{l}^{\prime}(x)-m \chi_{l}(x) \psi_{l}^{\prime}(m x)},
\end{array}\right.
$$

$\psi_{l}(x)=x j_{l}(x)$ и $\chi_{l}(x)=x h_{l}^{(1)}(x)-$ функции РиккатиБесселя, $h_{l}^{(1)}(x)$ - функция Ханкеля 1-го рода, нижний индекс означает порядок функций. Численный расчет коэффициентов, входящих в уравнения (32) и (33), проводится по методам, описанным в [52]. Отметим, что коэффициенты $A_{l l^{\prime}}=0$ при нечетном значении разности $l-l^{\prime}$, а коэффициенты $B_{l l^{\prime}}=0$ при четном значении $l-l^{\prime}$.

Если поляризация падающего света является правосторонней круговой $\left(\hat{\boldsymbol{\varepsilon}}_{0}=(\hat{\mathbf{x}}+i \hat{\mathbf{y}}) / \sqrt{2}\right)$, то коэффициенты $b_{l M(E)}^{(d)}=0$, т. е. $b_{l M(E)}^{(o)}=b_{l M(E)}^{(e)}$. Из полной системы уравнений (32), (33) остаются только уравнения (32). Обозначим их решение как $b_{l M R}^{(s)}$ и $b_{l E R}^{(s)}$. Если падающий свет поляризован по левому кругу $\left(\hat{\boldsymbol{\varepsilon}}_{0}=(\hat{\mathbf{x}}-i \hat{\mathbf{y}}) / \sqrt{2}\right)$, то нулю равны коэффициенты $b_{l M(E)}^{(s)}$ и соответственно $b_{l M(E)}^{(o)}=-b_{l M(E)}^{(e)}$. Решение оставшейся системы уравнений (33) обозначим как $b_{l M L}^{(d)}$ и $b_{l E L}^{(d)}$. Поскольку коэффициенты $A_{l l^{\prime}}=0$ при нечетном значении разности $l-l^{\prime}$, а коэффициенты $B_{l l^{\prime}}=0$ при четном значении $l-l^{\prime}$, то системы для правосторонней и левосторонней круговых поляризаций идентичны и их решения совпадают: $b_{l M R}^{(s)}=b_{l M L}^{(d)}=b_{l M}$ и $b_{l E R}^{(s)}=b_{l E L}^{(d)}=b_{l E}$.

Сделаем замену переменных в системах уравнений (32) и (33) для произвольной поляризации:

$$
\begin{aligned}
& b_{l M(E)}^{(s)}=\frac{1}{\sqrt{2}}\left(\varepsilon_{x}-i \varepsilon_{y}\right) b_{l M(E)}, \\
& b_{l M(E)}^{(d)}=\frac{1}{\sqrt{2}}\left(\varepsilon_{x}+i \varepsilon_{y}\right) b_{l M(E)} .
\end{aligned}
$$

При этом системы (32) и (33) идентичны и независимы от вектора поляризации падающей волны. Их решения $b_{l M(E)}$ позволяют находить коэффициенты $b_{l M(E)}^{(s)}$ и $b_{l M(E)}^{(d)}$ для любой поляризации по формулам (38), (39). C использованием новых переменных формулы для коэффициентов когерентного пропускания (23), отражения (24) и интенсивности некогерентно рассеянного поля (26) преобразуются:

$$
\begin{aligned}
& T_{c}=\left|1-\frac{\eta}{\sqrt{2} x^{2}} \sum_{l=1}^{\infty}(2 l+1)\left(b_{l M}+b_{l E}\right)\right|^{2}, \\
& R_{c}=\left|\frac{\eta}{\sqrt{2} x^{2}} \sum_{l=1}^{\infty}(-1)^{l}(2 l+1)\left(b_{l M}-b_{l E}\right)\right|^{2},
\end{aligned}
$$

$$
\begin{aligned}
& I_{i n c}^{r d}=\frac{\eta S_{2}(2 x \sin \theta)}{2 \pi x^{2} r^{2}}\left\{\left|\varepsilon_{x} \cos \varphi+\varepsilon_{y} \sin \varphi\right|^{2}\right. \\
& \times\left|\sum_{l=1}^{\infty} \frac{(2 l+1)}{l(l+1)}\left(\pi_{l}^{(1)}(\mu) b_{l M}+\tau_{l}^{(1)} b_{l E}\right)\right|^{2} \\
& +\left|\varepsilon_{x} \sin \varphi-\varepsilon_{y} \cos \varphi\right|^{2} \\
& \left.\times\left|\sum_{l=1}^{\infty} \frac{(2 l+1)}{l(l+1)}\left(\tau_{l}^{(1)}(\mu) b_{l M}+\pi_{l}^{(1)} b_{l E}\right)\right|^{2}\right\}
\end{aligned}
$$

Очевидно, что коэффициенты когерентного пропускания и отражения монослоя не зависят от состояния поляризации падающего излучения. В отличие от этого угловое распределение интенсивности некогерентно рассеянного излучения определяется компонентами вектора поляризации $\varepsilon_{x}$ и $\varepsilon_{y}$. Однако для коэффициента некогерентного рассеяния $F_{\text {inc }}$ эта зависимость исчезает вследствие интегрирования $I_{i n c}^{r d}(42)$ по азимутальному углу рассеяния $\varphi$ :

$$
\begin{aligned}
& F_{\text {inc }}=\frac{\eta}{2 x^{2}} \int_{0}^{\pi} S_{2}(2 x \sin \theta) \sin \theta \\
& \times\left\{\left|\sum_{l=1}^{\infty} \frac{(2 l+1)}{l(l+1)}\left(\pi_{l}^{(1)}(\mu) b_{l M}+\tau_{l}^{(1)}(\mu) b_{l E}\right)\right|^{2}\right. \\
& \left.+\left|\sum_{l=1}^{\infty} \frac{(2 l+1)}{l(l+1)}\left(\tau_{l}^{(1)}(\mu) b_{l M}+\pi_{l}^{(1)}(\mu) b_{l E}\right)\right|^{2}\right\} d \theta .
\end{aligned}
$$

Особым случаем является облучение монослоя естественным светом, который представляет собой совокупность световых волн со всеми возможными направлениями колебаний электрического вектора $\mathbf{E}_{0}$. Эта совокупность статистически симметрична относительно направления освещения. Для получения соответствующих коэффициентов когерентного пропускания и отражения, углового распределения интенсивности некогерентного поля и коэффициента рассеяния усредним соотношения (40)-(43) по углу поляризации $\alpha\left(\varepsilon_{x}=\cos \alpha, \varepsilon_{y}=\sin \alpha\right)$ с равномерным распределением в пределах от 0 до $2 \pi$. Очевидно, что формулы (40), (41) и (43) не изменяются. При этом прямопрошедшее и зеркально отраженное излучение остается неполяризованным. Формула для углового распределения интенсивности некогерентного поля (42) переходит в

$$
\begin{aligned}
& I_{i n c}^{r d}=\frac{\eta S_{2}(2 x \sin \theta)}{2 x^{2} r^{2}} \\
& \quad \times\left\{\left|\sum_{l=1}^{\infty} \frac{(2 l+1)}{l(l+1)}\left(\pi_{l}^{(1)}(\mu) b_{l M}+\tau_{l}^{(1)}(\mu) b_{l E}\right)\right|^{2}\right. \\
& \left.+\left|\sum_{l=1}^{\infty} \frac{(2 l+1)}{l(l+1)}\left(\tau_{l}^{(1)}(\mu) b_{l M}+\pi_{l}^{(1)}(\mu) b_{l E}\right)\right|^{2}\right\} .
\end{aligned}
$$

Некогерентно рассеянное излучение является частично поляризованным. Угловое распределение степени линей- 

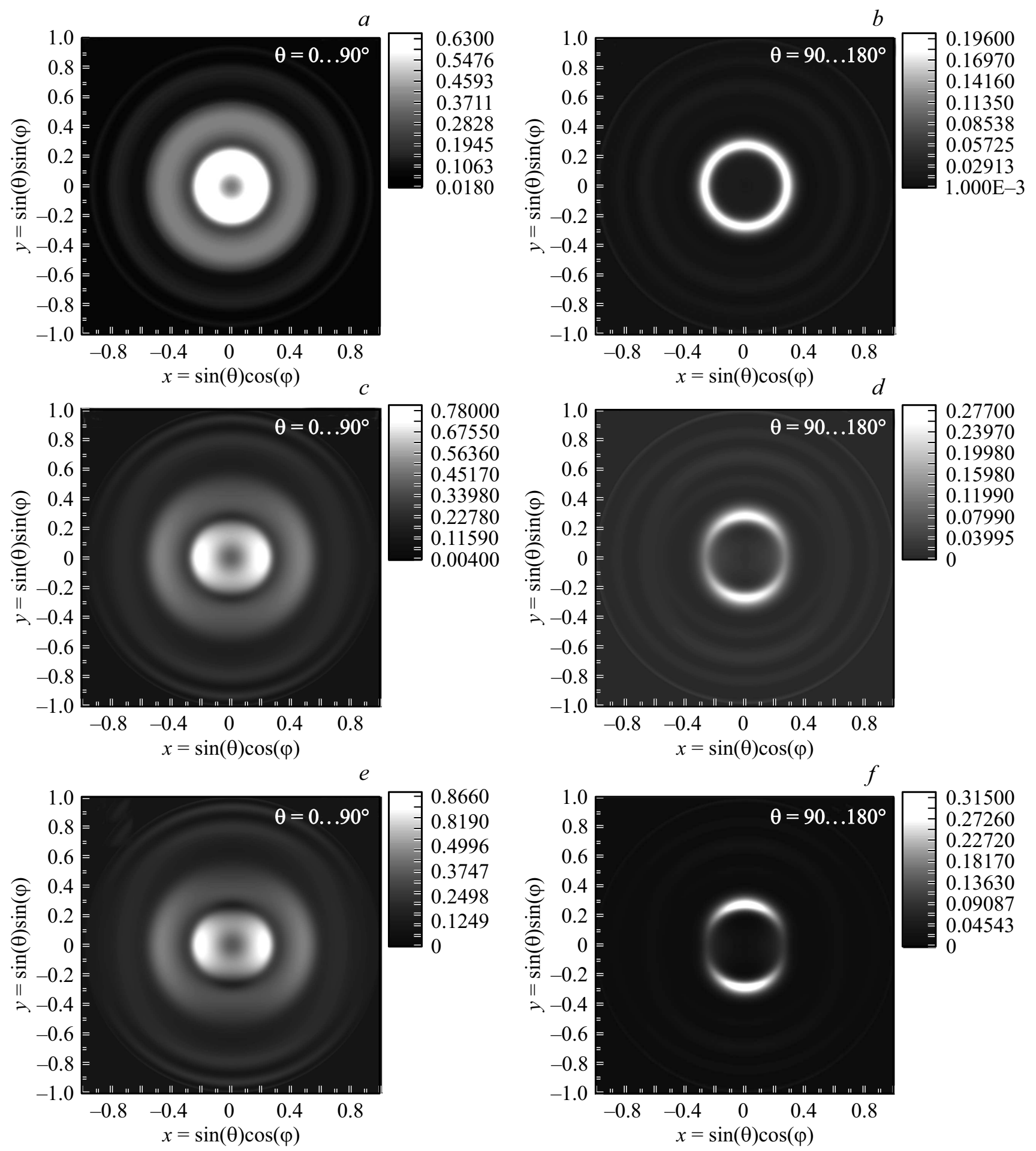

Рис. 3. Фотопредставления угловых зависимостей интенсивности $I_{i n c}^{r d}$ излучения, некогерентно рассеянного частично упорядоченным монослоем при циркулярной $\left(\varepsilon_{x}=0.7071+0 i, \varepsilon_{y}=0+0.7071 i\right)(a, b)$, эллиптической $\left(\varepsilon_{x}=0.9166+0 i, \varepsilon_{y}=0+0.4 i\right)(c, d)$ и линейной $\left(\varepsilon_{x}=1+0 i, \varepsilon_{y}=0+0 i\right)(e, f)$ поляризациях падающей волны. Левый столбец - рассеяние в переднюю полусферу, центр рисунков соответствует $\theta=0^{\circ}$, правый столбец - рассеяние в заднюю полусферу, центр рисунков соответствует $\theta=180^{\circ}$. Длина волны падающего излучения $\lambda=0.3 \mu \mathrm{m}$, диаметр частиц $D=1 \mu \mathrm{m}$, показатель преломления частиц $m=1.6+0 i$, фактор заполнения монослоя $\eta=0.5$. 

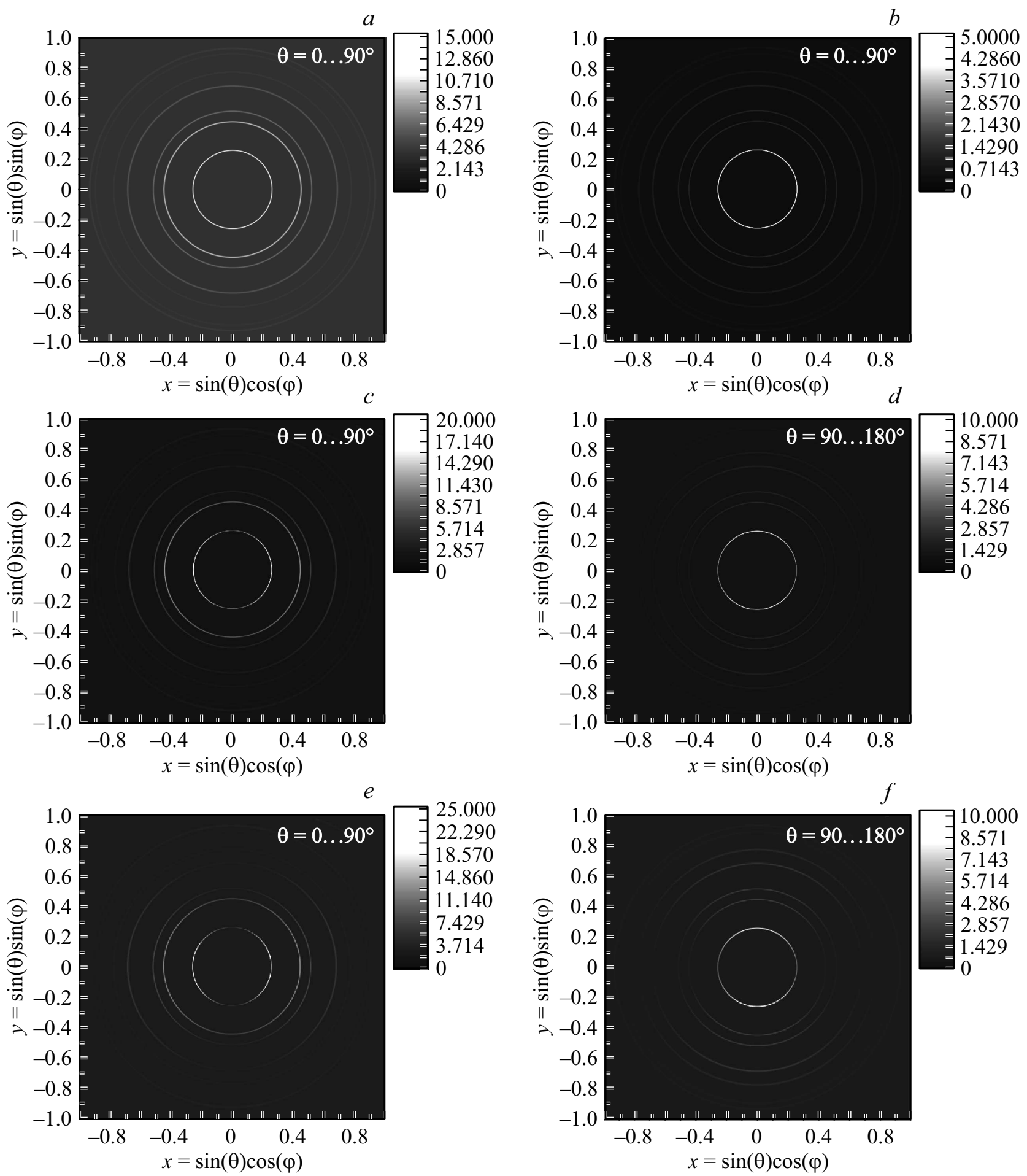

Рис. 4. Фотопредставления угловых зависимостей интенсивности $I_{i n c}^{r d}$ излучения, некогерентно рассеянного монослоем с неидеальной треугольной решеткой при циркулярной $\left(\varepsilon_{x}=0.7071+0 i, \varepsilon_{y}=0+0.7071 i\right)(a, b)$, эллиптической $\left(\varepsilon_{x}=0.9166+0 i\right.$, $\left.\varepsilon_{y}=0+0.4 i\right)(c, d)$ и линейной $\left(\varepsilon_{x}=1+0 i, \varepsilon_{y}=0+0 i\right)(e, f)$ поляризациях падающей волны. Левый столбец - рассеяние в переднюю полусферу, центр рисунков соответствует $\theta=0^{\circ}$, правый столбец - рассеяние в заднюю полусферу, центр рисунков соответствует $\theta=180^{\circ}$. Параметры РФР: $\sigma_{0}=0.01, a=b=0.5$ (длина корреляции $l_{c}=220[46,47]$ ). $\lambda=0.3 \mu \mathrm{m}, D=1 \mu \mathrm{m}$, $m=1.6+0 i, \eta=0.5$. 

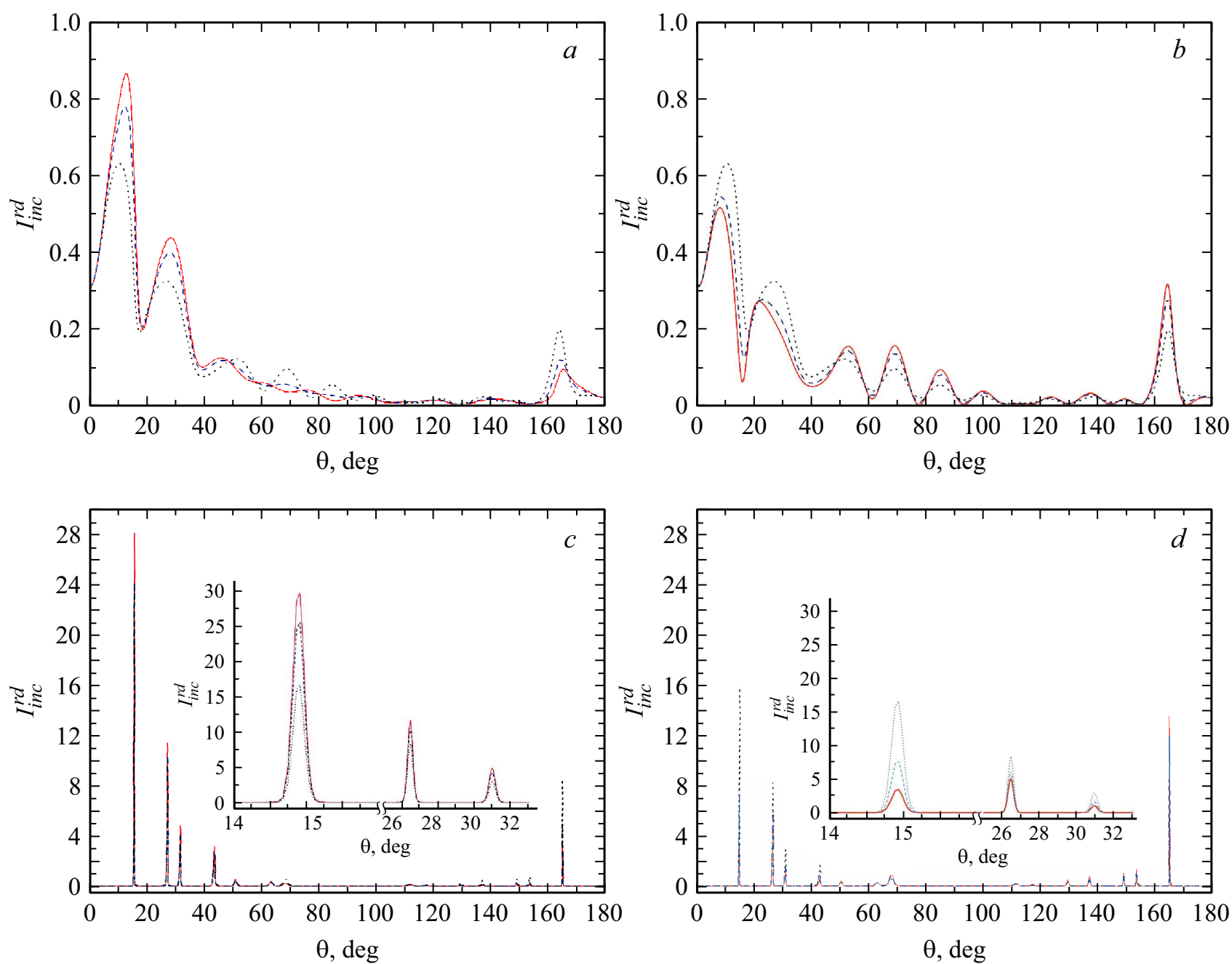

$\theta$, deg

$\cdots \cdots \varepsilon_{x}=(0.7071+0 i), \varepsilon_{y}=(0+0.7071 i)$ (circ. polariz.)

$\varepsilon_{x}=(0.9166+0 i), \varepsilon_{y}=(0+0.4 i)$ (ell. polariz.)

$-\varepsilon_{x}=(1+0 i), \varepsilon_{y}=(0+0 i)$ (lin. polariz)

Рис. 5. Зависимости интенсивности $I_{i n c}^{r d}$ излучения, некогерентно рассеянного частично упорядоченным монослоем $(a, b)$ и монослоем с неидеальной треугольной решеткой $(c, d)$, от полярного угла рассеяния $\theta$ при азимутальных углах рассеяния $\varphi \approx 0^{\circ}(a, c)$ и $\varphi \approx 90^{\circ}(b, d)$. На вставках рисунков $c, d$ первые 3 пика зависимости $I_{i n c}^{r d}$, соответствующие первому, второму и третьему дифракционным порядкам, показаны в более крупном масштабе. Параметры РФР: $\sigma_{0}=0.01, a=b=0.5(c, d)$. $\lambda=0.3 \mu \mathrm{m}, D=1 \mu \mathrm{m}, m=1.6+0 i, \eta=0.5$.

ной поляризации $P_{\text {inc }}\left(P_{\text {inc }}=\left(I_{\theta}-I_{\varphi}\right) /\left(I_{\theta}+I_{\varphi}\right)\right)$, где $I_{\theta}$ и $I_{\varphi}$ - интенсивности $\theta$ - и $\varphi$-компонент рассеянного излучения) определяется следующим образом:

$$
\begin{gathered}
P_{\text {inc }}(\theta)=\frac{\left\{\left|\sum_{l=1}^{\infty} \frac{(2 l+1)}{l(l+1)}\left(\pi_{l}^{(1)}(\mu) b_{l M}+\tau_{l}^{(1)}(\mu) b_{l E}\right)\right|^{2}\right.}{\left\{\left.\left|\sum_{l=1}^{\infty} \frac{(2 l+1)}{l(l+1)}\left(\tau_{l}^{(1)}(\mu) b_{l M}+\pi_{l}^{(1)}(\mu) b_{l E}\right)\right|^{2} \frac{(2 l+1)}{l(l+1)}\left(\pi_{l}^{(1)}(\mu) b_{l M}+\tau_{l}^{(1)}(\mu) b_{l E}\right)\right|^{2}\right.} . \\
+\left|\sum_{l=1}^{\infty} \frac{(2 l+1)}{l(l+1)}\left(\tau_{l}^{(1)}(\mu) b_{l M}+\pi_{l}^{(1)}(\mu) b_{l E}\right)\right|^{2}
\end{gathered}
$$

\section{Результаты расчетов}

Как уже было отмечено в предыдущем разделе, коэффициенты когерентного пропускания $T_{c}$, отражения $R_{c}$ и некогерентного рассеяния $F_{\text {inc }}$ не зависят от поляризации падающего излучения. Спектральные зависимости этих коэффициентов для частично упорядоченного монослоя и монослоя с неидеальной треугольной решеткой приведены в работах [51,52]. В данном разделе мы приводим результаты расчетов интенсивности $I_{i n c}^{r d}$ некогерентно рассеянного излучения в зависимости от состояния поляризации падающей волны. Расчеты выполнены по формулам (26), (27). 

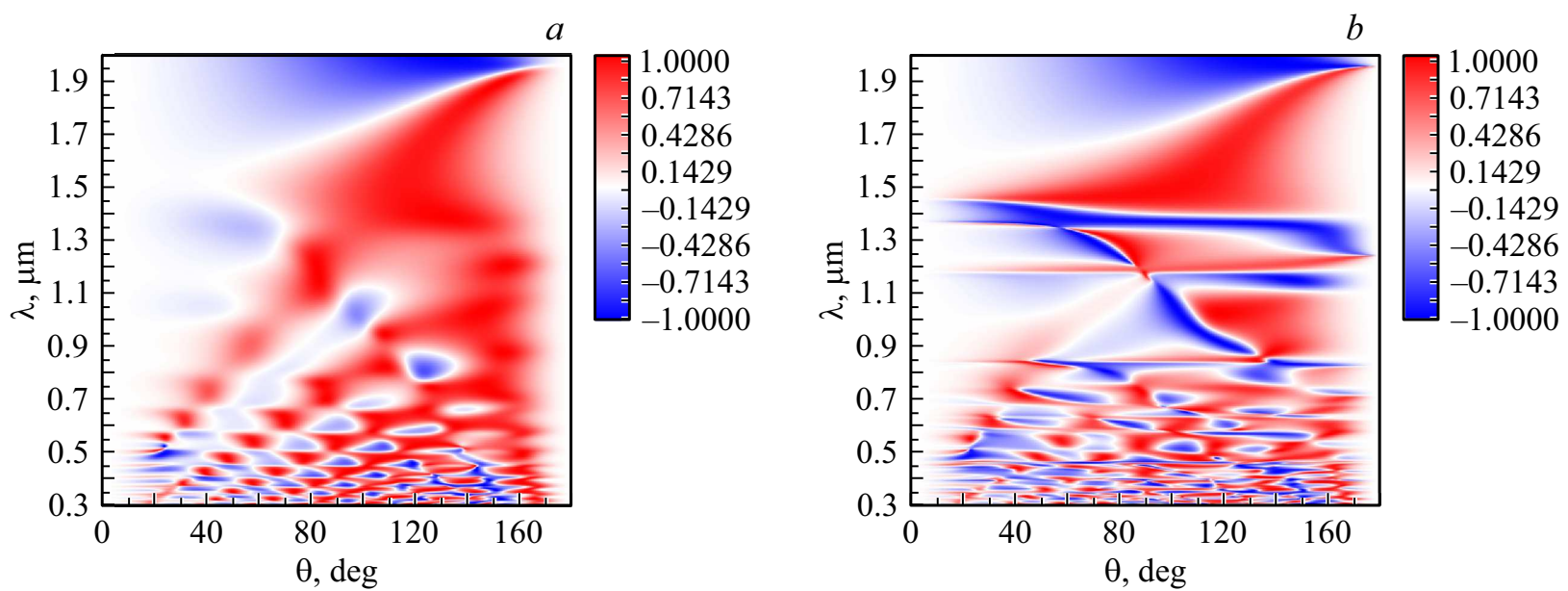

Рис. 6. Зависимости степени линейной поляризации $P_{\text {inc }}$ от длины волны $\lambda$ падающего излучения и полярного угла рассеяния $\theta$ для частично упорядоченного монослоя $(a)$ и монослоя с неидеальной треугольной решеткой $\left(\sigma_{0}=0.01, a=b=0.5\right)(b)$. $D=1 \mu \mathrm{m}, m=1.6+0 i, \eta=0.5$.

Структура монослоя (т. е. пространственное распределение частиц) задавалась с помощью радиальной функции распределения $g(u)$ (входящей в выражение (36)), описывающей вероятность нахождения центра частицы на расстоянии $u$ в плоскости монослоя относительно начала координат, находящегося в центре другой частицы. Радиальная функция распределения для частично упорядоченного монослоя (рис. $1, a$ ) рассчитывалась в приближении Перкуса-Йевика [45,53], а для монослоя с неидеальной решеткой — по формуле $[1,46,47]$

$$
g(u)=\rho_{0}^{-1} \sum_{i} \frac{N_{i}}{2 \pi u_{i}} \frac{1}{\sqrt{2 \pi} \sigma(u)} \exp \left(-\frac{\left(u-u_{i}\right)^{2}}{2 \sigma^{2}(u)}\right),
$$

где $N_{i}$ - число центров частиц, находящихся на координационной окружности с радиусом $u_{i}$ идеального кристалла,

$$
\sigma(u)=\sigma_{0}(a u+b)
$$

- функция размытия, описывающая уширение координационных окружностей. Параметры $\sigma_{0}, a$ и $b$ определяют степень упорядоченности монослоя частиц и расстояние, на котором РФР сходится к единице.

Отметим, что при использовании радиальных, а не двухчастичных [44,53,57] функций распределения для моделирования структуры монослоя предполагается равномерное распределение центров частиц по азимуту. В случае монослоя с неидеальной решеткой такое представление его структуры описывает двумерный поликристалл, для которого угловое распределение рассеянного излучения имеет вид выраженных тонких дифракционных колец, а не отдельных дифракционных пиков, характерных для монокристалла.

На рис. 3 показаны угловые зависимости интенсивности $I_{i n c}^{r d}$ излучения, некогерентно рассеянного частично упорядоченным монослоем (рис. 1, $a$ ) при циркулярной, эллиптической и линейной поляризациях падающей волны. На рис. 4 показаны аналогичные зависимости для монослоя с неидеальной треугольной решеткой (рис. $1, b$ ). Можно видеть, что при освещении циркулярно поляризованным излучением (рис. $3, a, b$, рис. $4, a, b)$ угловые распределения не зависят от азимутального угла рассеяния $\varphi$, а при освещении эллиптически (рис. $3, c, d$, рис. $4, c, d$ ) и линейно (рис. $3, e, f$, рис. $4, e, f$ ) поляризованным - зависят.

На рис. 5 показаны зависимости интенсивности $I_{\text {inc }}^{r d}(\theta)$ при азимутальных углах рассеяния $\varphi \approx 0^{\circ}$ и $\varphi \approx 90^{\circ}$.

Из рис. 3-5 видно, что большая часть энергии рассеянного излучения сосредоточена в пиках, близких к $\theta=0^{\circ}$ и $\theta=180^{\circ}$ (на рис. 3 и 4 это светлые кольца, наиболее близкие к центрам рисунков). Поляризация падающей волны заметно влияет на амплитуду пиков (рис. 5) и распределение интенсивности по азимутальному углу рассеяния (рис. 3, 4). В частности, при освещении монослоя волной с эллиптической и линейной поляризацией максимумы интенсивности для одних и тех же дифракционных порядков „поворачиваются“ на $90^{\circ}$ по углу $\varphi$ при рассеянии в заднюю полусферу по сравнению с их значениями для передней полусферы (рис. 3, 4). Как видно из рис. $5, c, d$, практически вся энергия излучения, рассеянного упорядоченным монослоем, сосредоточена в дифракционных пиках (кольцах), угловое положение (по углу $\theta$ ) которых практически не зависит от поляризации падающей волны. Для частично упорядоченного монослоя (рис. 5, $a, b$ ) характерно размытие пиков и угловое смещение их максимумов в зависимости от состояния поляризации падающей волны. Величина некогерентной составляющей в направлении распространения падающей волны увеличивается с ростом разупорядоченности слоя.

На рис. 6 показаны угловые и спектральные зависимости степени линейной поляризации $P_{\text {inc }}$ для частично 
упорядоченного монослоя и монослоя с неидеальной треугольной решеткой, рассчитанные по формуле (45).

Тип пространственной упорядоченности монослоя сильно влияет на величину $P_{\text {inc }}$ в области длин волн, сопоставимых с диаметром частиц. Наиболее резкое изменение $P_{\text {inc }}$ имеет место в областях резонансов для монослоя с треугольной решеткой (на рис. $6, b$ области длин волн $\lambda=0.8-0.9,1.1-1.45 \mu \mathrm{m})$.

\section{Заключение}

Разработанный нами метод [51,52] определения оптических характеристик монослоя однородных монодисперсных сферических частиц, освещаемого по нормали плоской циркулярно поляризованной электромагнитной волной, основанный на ККП теории многократного рассеяния, обобщен на случай произвольной поляризации падающей волны. Получены формулы для описания некогерентного рассеяния, учитывающие многократное рассеяние в слое и состояние поляризации падающего излучения. Приведены результаты, иллюстрирующие влияние поляризации падающей волны на угловую структуру рассеянного излучения (поляризации когерентно прошедшего и когерентно отраженного полей сохраняются).

Рассчитаны и проанализированы спектральные и угловые зависимости степени линейной поляризации рассеянного излучения при освещении монослоя естественным светом. Показано, что тип пространственной организации частиц оказывает сильное влияние на эти зависимости. Наиболее резкое изменение степени линейной поляризации имеет место в областях резонансов, обусловленных большой степенью упорядоченности монослоя с „неидеальным дальним“ порядком.

Полученные результаты могут быть использованы при решении задач фотоники и оптоэлектроники, разработке и создании дисплеев, фотонных кристаллов, поляризаторов и других устройств на основе монослоев частиц.

Работа поддержана Белорусским республиканским фондом фундаментальных исследований (БРФФИ), проект Ф18РА-003. Авторы выражают благодарность сотруднику Института физики НАН Беларуси А.В. Конколовичу за полезные дискуссии.

\section{Список литературы}

[1] Loiko V.A., Miskevich A.A. // Multiple Light Scattering, Radiative Transfer and Remote Sensing. Springer Series in Light Scattering. V. 1. / Ed by Kokhanovsky A. Berlin: Springer, 2018. P. 101-230.

[2] Yamasaki T., Tsutsui T. // Jpn. J. Appl. Phys. 1999. V. 38. P. 5916.

[3] Sun W., Videen G., Lin B. // Appl. Opt. 2007. V. 46. P. 1150.

[4] Wang B., Jin Y., He S. // J. Appl. Phys. 2009. V. 106. P. 014508 .
[5] Nayak B.K., Sun K., Rothenbach Ch., Gupta M.C. // Appl. Opt. 2011. V. 50. P. 2349.

[6] Fujii G., Matsumoto T., Takahashi T. // Opt. Exp. 2012. V. 20. P. 7300.

[7] Wu X.H., Yamilov A., Noh H., Cao H., Seelig E.W., Chang R.P.H. // J. Opt. Soc. B. 2004. V. 21. P. 159.

[8] Rho Y., Wanit M., Yeo J., Hong S., Han S., Choi J.-H., Hong W.-H., Lee D., Ko S.H. // J. Phys. D: Appl. Phys. 2013. V. 46. P. 024006.

[9] Wiersma D.S. // Nat. Photon. 2013. V. 7. P. 188.

[10] Kim I., Jeong D.S., Lee W.S., Kim W.M., Lee T.-S., Lee D.K., Song J.-H., Kim J.-K., Lee K.-S. // Opt. Exp. 2014. V. 22. P. A1431.

[11] Лойко В.А., Мискевич А.А. // Опт. и спектр. 2013. Т. 115. C. 316.

[12] Miskevich A.A., Loiko V.A. // J. Quant. Spect. Rad. Transf. 2014. V. 136. P. 58.

[13] Miskevich A.A., Loiko V.A. // J. Quant. Spect. Rad. Transf. 2014. V. 146. P. 355.

[14] Miskevich A.A., Loiko V.A. // J. Quant. Spect. Rad. Transf. 2015. V. 167. P. 23.

[15] Лойко В.А., Мискевич А.А. // Опт. и спектр. 2017. Т. 122. C. 825.

[16] Ohtaka K. // J. Phys. C: Solid St. Phys. 1980. V. 13. P. 667.

[17] Ohtaka K., Suda Y., Nagano S. // Phys. Rev. B. 2000. V. 61. P. 5267.

[18] Modinos A. // Physica A. 1987. V. 141. P. 575.

[19] Stefanou N., Modinos A. // J. Phys.: Condens. Matter. 1991. V. 3. P. 8135.

[20] Stefanou N., Modinos A. // J. Phys.: Condens. Matter. 1993. V. 5. P. 8859.

[21] Foldy L.L. // Phys. Rev. 1945. V. 67. P. 107.

[22] Lax M. // Rev. Mod. Phys. 1951. V. 23. P. 287.

[23] Lax M. // Phys. Rev. 1952. V. 85. P. 621.

[24] Twersky V. // J. Appl. Phys. 1952. V. 23. P. 407.

[25] Twersky V. // J. Math. Phys. 1975. V. 16. P. 633.

[26] Fikioris J.G., Waterman P.C. // J. Math. Phys. 1964. V. 5. P. 1413; JQSRT. 2013. V. 123. P. 8.

[27] Mathur N.C., Yeh K.C. // J. Math. Phys. 1964. V. 5. P. 1619.

[28] Varadan V.V., Varadan V.K. // Phys. Rev. D. 1980. V. 21. P. 388.

[29] Tsang L., Kong J.A. // Radio Sci. 1983. V. 18. P. 1260.

[30] Tsang L., Chen C.-T., Chang A.T.C., Guo J., Ding K.-H. // Radio Sci. 2000. V. 35. P. 731.

[31] Hong K.M. // J. Opt. Soc. Am. 1980. V. 70. P. 821.

[32] Mackowski D. // Proc. R. Soc. Lond. A. 1991. V. 433. P. 599; JOSA A. 1994. V. 11. P. 2851.

[33] Xu Y. // Appl. Opt. 1995. V. 34. P. 4573; Phys. Lett. A. 1998. V. 249. P. 30.

[34] Верещагин В.Г., Понявина А.Н., Сильванович Н.И. // Докл. АН БССР. 1990. Т. 34. С. 123.

[35] Ponyavina A.N., Kachan S.M., Silvanovich N.I. // JOSA B. 2004. V. 21. P. 1866.

[36] Loiko V., Molochko V. // Part. Part. Syst. Charact. 1996. V. 13. P. 227.

[37] Loiko V.A., Dick V.P., Ivanov A.P. // J. Opt. Soc. Am. A. 2000. V. 17. P. 2040.

[38] Loiko V.A., Miskevich A.A. // Appl. Opt. 2005. V. 44. P. 3759.

[39] Лойко В.А., Мискевич А.А. // Опт. и спектр. 2005. Т. 98. C. 65 .

[40] Mishchenko M.I., Liu L., Mackowski D.W., Cairns B., Videen G. // Opt. Exp. 2007. V. 15. P. 2822. 
[41] Mishchenko M.I., Dlugach J.M., Mackowski D.W. // JOSA A. 2018. V. 33. P. 2144.

[42] Okada Y., Kokhanovsky A.A. // J. Quant. Spectr. Rad. Transf. 2009. V. 110. P. 902.

[43] Garc?a-Valenzuela A., Guti?rrez-Reyes E., Barrera R. // JOSA A. 2012. V. 29. P. 1161

[44] Ziman J. Models of Disorder. Cambridge: Univ. Press, 1979. $525 \mathrm{p}$.

[45] Percus J.K., Yevick G.J. // Phys. Rev. 1958. V. 110. P. 1.

[46] Miskevich A.A., Loiko V.A. // J. Quant. Spectr. Rad. Transf. 2011. V. 112. P. 1082.

[47] Мискевич А.А., Лойко В.А. // ЖЭТФ. 2011. Т. 140. С. 5.

[48] Miskevich A.A., Loiko V.A. // Nanosyst.: Phys., Chem., Math. 2013. V. 4. P. 778.

[49] Мискевич А.А., Лойко В.А. // ЖЭТФ. 2014. Т. 146. С. 246.

[50] Miskevich A.A., Loiko V.A. // J. Quant. Spectr. Rad. Transf. 2015. V. 151. P. 260.

[51] Loiko N.A., Miskevich A.A., Loiko V.A. // J. Opt. Soc. Am. A. 2018. V. 35. P. 108.

[52] Лойко Н.А., Мискевич А.А., Лойко В.А. // ЖЭТФ. 2018. T. 153. C. 193.

[53] Иванов А.П., Лойко В.А., Дик В.П. Распространение света в плотноупакованных дисперсных средах. Минск: Наука и техника, 1988. $191 \mathrm{c.}$

[54] Morse P.M., Feshbach H. Methods of Theoretical Physics. NY: McGraw-Hill Book, 1953.

[55] Babenko V.A., Astafyeva L.G., Kuzmin V.N. Electromagnetic Scattering in Disperse Media: Inhomogeneous and Anisotropic Particles. Berlin: Springer, 2003. 434 p.

[56] Mishchenko M.I., Travis L.D., Lacis A.A. Scattering, Absorption, and Emission of Light by Small Particles. Cambridge: Univ. Press, 2002. 445 p. 